\title{
XMM\#Newton Observations of the Red Type 1.8 Quasar 2M1049+5837: Reflection from Cold and Warm (lonized) Matter
}

\section{Citation}

Wilkes, B. J., K. A. Pounds, and G. D. Schmidt. 2008. “XMM\#Newton Observations of the Red Type 1.8 Quasar 2M1049+5837: Reflection from Cold and Warm (lonized) Matter ." The Astrophysical Journal 680 (1) (June 10): 110-118. doi:10.1086/588039.

\section{Published Version}

doi:10.1086/588039

\section{Permanent link}

http://nrs.harvard.edu/urn-3:HUL.InstRepos:29921881

\section{Terms of Use}

This article was downloaded from Harvard University's DASH repository, and is made available under the terms and conditions applicable to Other Posted Material, as set forth at http:// nrs.harvard.edu/urn-3:HUL.InstRepos:dash.current.terms-of-use\#LAA

\section{Share Your Story}

The Harvard community has made this article openly available.

Please share how this access benefits you. Submit a story.

\section{Accessibility}




\title{
XMM-NEWTON OBSERVATIONS OF THE RED TYPE 1.8 QUASAR 2M1049+5837: REFLECTION FROM COLD AND WARM (IONIZED) MATTER
}

\author{
B. J. WiLKes, ${ }^{1}$ K. A. Pounds, ${ }^{2}$ ANd G. D. SchmidT ${ }^{3}$ \\ Received 2007 December 6; accepted 2008 March 3
}

\begin{abstract}
Chandra observations of the type 1.8, low-redshift $(z=0.115)$, red $\left(J-K_{S} \sim 2\right)$ quasar 2MASSJ 104943+583750 (2M1049+5837) indicated an unusually hard X-ray spectrum (HR $\sim 0.6$ ), suggesting obscuration of $N_{\mathrm{H}} \sim 4 \times 10^{22} \mathrm{~cm}^{-2}$ and a flat $\Gamma \sim 0.4$ power-law slope. A higher signal-to-noise ratio $X M M$-Newton observation reported here reveals a more complex spectrum, being extremely hard above $\sim 2 \mathrm{keV}, \Gamma \sim-0.6$, with a well-defined soft excess similar to the bright, nearby Seyfert 2 galaxy Mrk 3. Such extremely hard quasar spectra cannot be a dominant contributor to the cosmic X-ray background (CXRB; $\Gamma \sim 1.4$ ). Modeling of $2 \mathrm{M} 1049+5837$ shows the observed $2-10 \mathrm{keV}$ spectrum to be the sum of a strongly absorbed "normal" $\Gamma \sim 1.8$ underlying quasar X-ray continuum, and a cold reflection component $(R \sim 2)$. The strong attenuation of the intrinsic X-ray continuum by a sub-Compton-thick line-of-sight column, $N_{\mathrm{H}} \sim 3.4 \times 10^{23} \mathrm{~cm}^{-2}$, reveals a soft X-ray emission component whose spectrum indicates reprocessing/ emission from, possibly extended, photoionized gas. The luminosity of the soft X-ray component is similar to Seyfert 2 galaxies, an order of magnitude less than comparable type 1 AGNs, suggesting partial obscuration of core-bright emission. The optical emission is complex, with distinct red and blue scattered light components believed to originate in two extended regions visible in $H S T$ imaging data. The unusual combination of properties: optical and X-ray obscuration of the nuclear emission, partially obscured broad but unobscured narrow emission lines, and partially obscured soft X-ray excess, implies an intermediate viewing angle, over or through the edge of an obscuring disk + wind or torus. 2M1049+5837 again demonstrates both the ambiguity of low signal-to-noise X-ray spectra/hardness ratios and the potential of 2MASS-selected red sources for exploring the complex obscuration and geometry of the nuclear regions in AGNs.
\end{abstract}

Subject headings: quasars: individual (2MASS J1049+5837) — X-rays: galaxies

\section{INTRODUCTION}

The strong dependence of their multiwavelength appearance on the inclination to the line of sight is both a fascinating and frustrating feature of active galactic nuclei (AGNs). As a result, the subset of the population being studied depends strongly on the selection techniques and wave bands used to find them. When combined with their emission over the full range of the electromagnetic spectrum, and the likelihood that their appearance also depends on their age, black hole mass, luminosity, etc., obtaining an accurate view of the parent population is a challenge.

The big advances in observing capability over the past $5-$ 10 years have facilitated large, multiwavelength surveys which allow us to compare and combine selection methods across different wave bands, as well as to push deeper to sample more of the population. These surveys have found new subsets of AGNs whose relation/importance to the population as a whole remain to be determined.

One such subset are the AGNs found in the Two Micron All Sky Survey (2MASS) database by virtue of their red $J-K_{S}$ color ( $>2$; Cutri et al. 2002). The resulting sample of 2MASS sources is dominated by new AGNs/quasars characterized by red colors throughout the optical and near-infrared (IR), and generally having broad lines (type 1 /type $2 \sim 3$ ). The sample includes a significant number of transition objects: type 1.2-1.9; have hard X-ray emission indicating $\log N_{\mathrm{H}} \sim 22$ (Wilkes et al. 2002, 2005); and high optical polarization ( $>3 \%$; Smith et al. 2002, 2003). At

\footnotetext{
1 Harvard-Smithsonian Center for Astrophysics, Cambridge, MA 02138.

2 Department of Physics and Astronomy, University of Leicester, Leicester LE1 7RH, UK

Steward Observatory, University of Arizona, Tucson, AZ 85721.
}

low redshift they may represent as much as $20 \%$ of the AGN population and likely comprise a significant subset of the moderately obscured AGNs required by current cosmic X-ray background (CXRB) models (Gilli et al. 2007). The high-redshift population of such sources is not yet known due to the combination of the bright magnitude limit of the 2MASS survey and the lower efficiency of the near-IR selection as the optical emission shifts through the observed wave bands. Spitzer surveys are finding many red AGNs with comparable or steeper spectral slopes in the mid-IR which may represent high-redshift counterparts of the 2MASS-selected ones (Polletta et al. 2006).

The nuclear absorbing columns seen in the red, 2MASS AGNs are often intermediate between those typically found for type 1 and type $2 \mathrm{AGNs} /$ quasars. When combined with their range of optical types, this suggests that red, 2MASS AGNs are intermediate sources, perhaps with a very specific line of sight, e.g., through the edge of a torus or disk + wind. Alternatives include a short-term state in the evolution of AGNs as obscuring material is blown away from the nuclear regions or obscuration of a different nature, such as through an edge-on host galaxy or unusually thick dust cloud. Studies of their spectral energy distributions (SEDs; Kuraszkiewicz et al. 2008) suggest that both near-nuclear and host galaxy obscuration play a role. The near-IR selection is efficient at finding these AGNs at low redshift, and the obscuration of direct AGN emission offers the potential to probe the nuclear regions away from the nuclear glare, providing the opportunity to learn more about the obscuring material responsible for the AGN classes.

Accordingly, we embarked on an X-ray study of a subset of the red, 2MASS AGNs with Chandra which suggested that substantial nuclear obscuration is generally present in these sources (Wilkes et al. 2002). We then followed up several of the brightest 
TABLE 1

XMM-Newton Observation Details

\begin{tabular}{cccccr}
\hline \hline Camera & 2 MASS & Date & Filter & Exposure & Counts \\
\hline pn..................... & $1049+5837$ & 2005 Oct 10 & Thin & $20.35 \mathrm{ks}$ & 1013 \\
MOS ................. & $1049+5837$ & 2005 Oct 10 & Thin & $55.20 \mathrm{~s}$ & 689 \\
\hline
\end{tabular}

and/or most unusual with XMM-Newton in order to obtain sufficient signal to better resolve the X-ray spectra. We found that the X-ray spectra are generally complex, with several emission and absorption components (Wilkes et al. 2005; Pounds et al. 2005). XMM-Newton observations of five red AGNs found a mean observed spectrum over the $2-10 \mathrm{keV}$ band that was hard, with a photon index $\Gamma \sim 1.4$ similar to that of the CXRB (Wilkes et al. 2005). In two cases, both optically type 2 , the spectral hardness was explained by observing a "canonical" $\Gamma \sim 1.8$ continuum through a large column $\left(N_{\mathrm{H}} \sim 10^{22} \mathrm{~cm}^{-2}\right)$ of cold gas. Of the other three cases, all broad-line AGNs, a substantial absorbing column was only evident in one, but all three XMM-Newton spectra showed soft X-ray excesses, possibly related to the optical scattering region (see also Pounds et al. 2005). Similar, obscured AGNs with complex X-ray spectra are being found in the Swift Burst Alert Telescope AGN survey, selected by their hard X-ray flux (14-495 keV; Ueda et al. 2007; Winter et al. 2008).

The type 1.8 quasar $2 \mathrm{M} 1049+5837(z=0.115)$, which was part of our deeper X-ray XMM-Newton follow-up program, is a particularly interesting and unusual source. It has unique optical polarization properties, including two scattered light components originating in two distinct regions (Schmidt et al. 2007). A blue scattered component probably originates in an extended "blue fan" of emission revealed by deep Hubble Space Telescope (HST) imaging, and a red component is most likely from a bipolar scatterer similar to those typically seen in polarized AGNs (Schmidt et al. 2007). The $X M M$-Newton data show an extremely hard spectrum, which can be explained by a combination of strong absorption of a direct power law and unobscured reflection. The strong obscuration of the direct emission allows the detection and quantification of a soft excess which is indicative of emission from photoionized gas. We present the XMM-Newton spectral analysis and its implications in terms of the AGN "unified scheme" and discuss the possible relationships between the various X-ray components and the multiwavelength properties of this unusual AGN.

\section{XMM-NEWTON OBSERVATIONS AND DATA ANALYSIS}

2M1049+5837 was observed by XMM-Newton on 2005 October 10 with an on-source exposure of $26.5 \mathrm{ks}$ (Table 1). X-ray data were available throughout each observation from the EPIC pn (Strüder et al. 2001) and MOS (Turner et al. 2001) cameras, providing moderate-resolution spectra over the energy band $\sim 0.3-10 \mathrm{keV}$.

The X-ray data were first screened with the $X M M$ SAS version 6.1 software and events corresponding to patterns $0-4$ (singleand double-pixel events) selected for the pn data, and patterns 0-12 for MOS1 and MOS2, the latter then being combined. A low-energy cut of $300 \mathrm{eV}$ was applied to all X-ray data, and known hot or bad pixels were removed. Source counts were obtained from a circular region of $45^{\prime \prime}$ radius centered on the target source, with the background being taken from a similar region offset from, but close to, the source. The integrated data set from each camera was then available for spectral analysis. Individual EPIC spectra were binned to a minimum of 20 counts bin ${ }^{-1}$ to facilitate use of the $\chi^{2}$ minimization technique in spectral fitting.

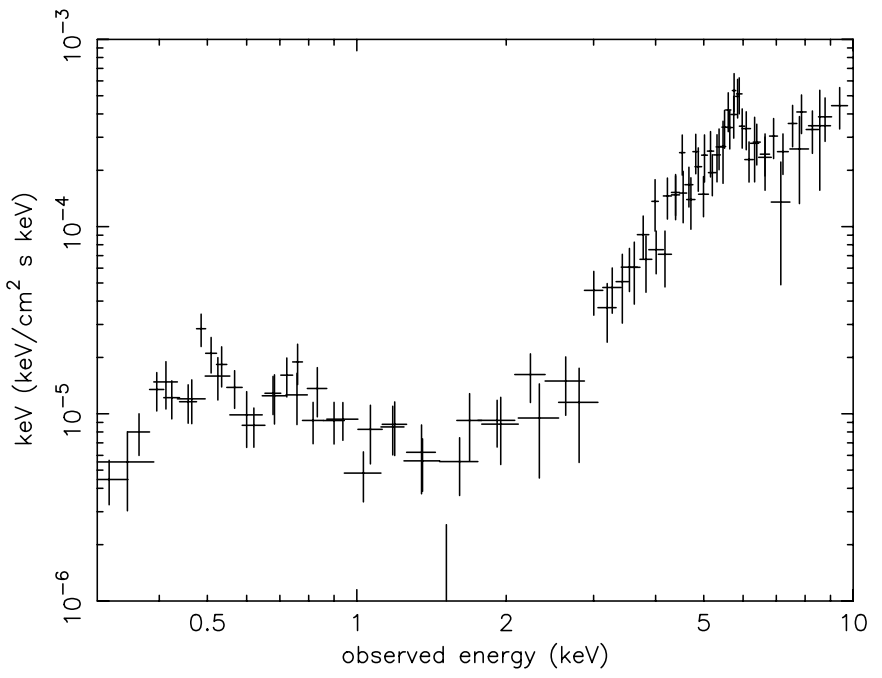

FIG. 1.-Fluxed spectrum of the integrated EPIC data from 2M1049+5837. The extremely hard spectrum of $2 \mathrm{M} 1049+5837$ above $\sim 2 \mathrm{keV}$ is evident in comparison with a typical Seyfert continuum, which would be almost flat on this plot. A strong Fe $\mathrm{K}$ emission line and spectral break below $\sim 2 \mathrm{keV}$ are also evident in the raw data.

Spectral fitting was based on the XSPEC package (Arnaud 1996), and all spectral fits include absorption due to the $2 \mathrm{M} 1049+5837$ line-of-sight Galactic column of $N_{\mathrm{H}}=6 \times 10^{19} \mathrm{~cm}^{-2}$ (Stark et al. 1992). Errors are quoted at the $90 \%$ confidence level $\left(\Delta \chi^{2}=2.7\right.$ for one interesting parameter). We assume $H_{0}=70 \mathrm{~km} \mathrm{~s}^{-1} \mathrm{Mpc}^{-1}$, $\Omega_{\Lambda}=0.7$, and $\Omega_{M}=0.3$.

\subsection{The Hard (2-10 keV) EPIC Spectrum}

Figure 1 shows the fluxed EPIC spectrum integrated over the full observation of $2 \mathrm{M} 1049+5837$. The spectrum is highly structured and unusually hard above $\sim 2 \mathrm{keV}$ (a typical unabsorbed type 1 Seyfert spectrum is almost flat in such a plot). A much softer spectral component dominates below $\sim 2 \mathrm{keV}$, with 2M1049+ 5837 remaining visible over the whole EPIC spectral range to $\sim 0.3 \mathrm{keV}$.

We initially restricted modeling of the EPIC data to the hard X-ray $(2-10 \mathrm{keV})$ band. A power-law fit above $2 \mathrm{keV}$ is statistically poor but confirmed the observed spectrum to be extremely hard, with a photon index $\Gamma \sim-0.6$. The $6 \mathrm{keV} \mathrm{Fe} \mathrm{K} \alpha$ emission line is also apparent. Only in the Seyfert 2 galaxy Mrk 3 among bright, nearby sources has such an extremely hard spectrum been observed (Pounds \& Page 2005). In that case, the hard X-ray spectrum is formed by a canonical Seyfert power law of photon index $\Gamma \sim 1.8$, attenuated by a near-Compton-thick absorber, together with a strong reflection component. An intense $\mathrm{Fe} \mathrm{K} \alpha$ line is consistent with fluorescence in the same matter responsible for the continuum reflection. Given the similarly unusual structure in the fluxed spectra, we tested the successful Mrk 3 spectral model with the XMM-Newton data for $2 \mathrm{M} 1049+5837$ over the $2-10 \mathrm{keV}$ spectral band.

In fitting this model to the EPIC data, the power-law and Fe K line parameters were initially left free, but tied for the pn and MOS fits. Cold reflection was modeled with pexrav in XSPEC (Magdziarz \& Zdziarski 1995), with a reflection factor $R(=\Omega$ / $2 \pi$, where $\Omega$ is the solid angle subtended by the reflector at the continuum source) left free, but common to both pn and MOS fits. The high-energy cutoff of the incident power law was set at $150 \mathrm{keV}$, and the viewing angle (to the reflector) at the default value of $60^{\circ}$. Element abundances were set to be solar. An excellent fit $\left(\chi^{2}=35\right.$ for 50 degrees of freedom [dof] $)$ was obtained for a 


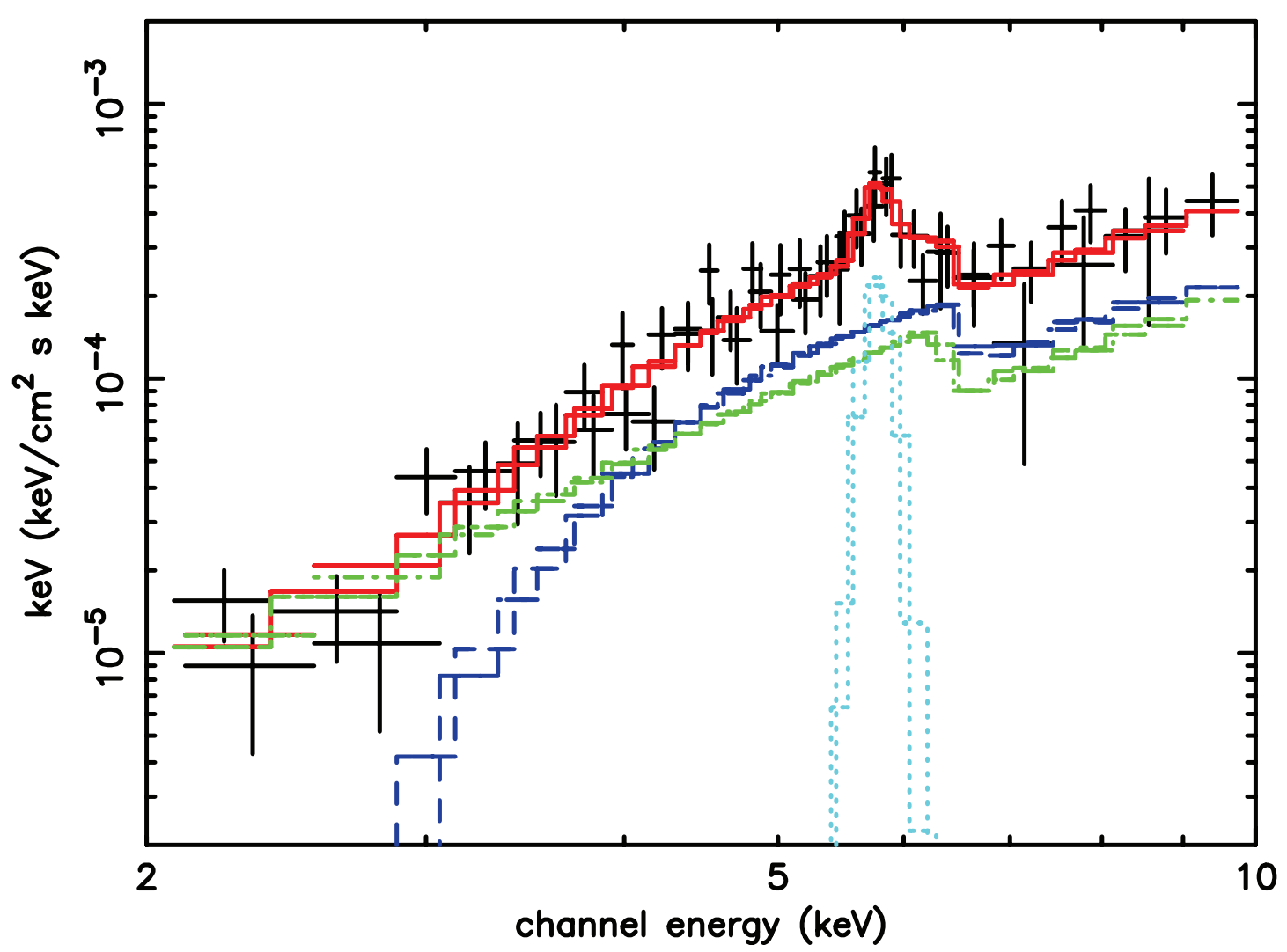

FIG. 2.-Model fit (red) to the EPIC data at 2-10 keV. The model components are an absorbed power law (dark blue), continuum reflection ( green), and Fe K fluorescence line (light blue).

(poorly constrained) photon index $\Gamma \sim 1.8$, attenuated by a column of cold gas of $N_{\mathrm{H}} \sim 4 \times 10^{23} \mathrm{~cm}^{-2}$. The strong reflection continuum was represented by $R=4 \pm 1$ (unphysical in this simple model). It is important to note that both absorption and reflection are required to model the observed continuum down to $2 \mathrm{keV}$; without the reflection component the model spectrum falls too steeply below $\sim 4 \mathrm{keV}$, yielding a significantly worse fit $(2-10 \mathrm{keV})$ of $\chi^{2}=67 / 51$.

The Fe K $\alpha$ emission line was well fitted by a Gaussian at $6.44 \pm$ $0.05 \mathrm{keV}$ (AGN rest frame), of width $\sigma=110 \pm 60 \mathrm{eV},{ }^{4}$ and flux $=(2 \pm 0.7) \times 10^{-6}$ photons $\mathrm{cm}^{-2} \mathrm{~s}^{-1}$. The line equivalent width (EW) is $540 \pm 170 \mathrm{eV}$, compared with the reflected continuum. (We note that this EW is significantly lower than the theoretical prediction of $\sim 1.2 \mathrm{keV}$ for reflection from optically thick matter with a solar abundance of Fe; Matt et al. 1997.)

This best-fit model for the $2-10 \mathrm{keV}$ spectrum of $2 \mathrm{M} 1049+$ 5837 is reproduced in Figure 2.

\subsection{The Soft X-Ray Emission}

Extending the hard X-ray (2-10 keV) model fit below $2 \mathrm{keV}$ (Fig. 3) reveals a clear "soft excess" ( $\chi^{2}=410$ for 86 dof $)$. With the hard X-ray parameters held fixed, the fit was improved, but remained statistically unacceptable $\left(\chi^{2}=191\right.$ for 92 dof $)$ after the addition of a second (unabsorbed) power-law component (Fig. 4), with photon index fixed to equal that of the primary hard X-ray power law. This second power-law component was intended to model X-ray flux scattered into the line of sight by electrons in the ionized gas perhaps also responsible for the polarized optical light (Schmidt et al. 2007). Visual examination

\footnotetext{
line.

${ }^{4}$ The signal-to-noise ratio of the data is insufficient to rule out an unresolved
}

of Figure 4 shows unmodeled spectral structure in $2 \mathrm{M} 1049+5837$, notably the flux peaks near $\sim 0.5$ and $\sim 0.8 \mathrm{keV}$, to be mainly responsible for the still unacceptable fit.

Again, the EPIC spectrum of Mrk 3 offered a valuable guide, wherein similar soft X-ray structure was ascribed to line emission from photoionized and photoexcited gas (Pounds \& Page 2005 and references therein). ${ }^{5}$ We therefore added a further component to the spectral model of $2 \mathrm{M} 1049+5837$ to represent line emission from ionized gas, modeled by an XSTAR grid (Kallman et al. 1996). Here the free parameters were the ionization parameter and emission measure, while the metal abundances (C-Fe) were initially fixed at their solar values. The fit was now acceptable $\left(\chi^{2}=76 / 75\right)$, with an ionization parameter $\xi\left(=L / n r^{2}\right)$ of $25 \pm$ 15 ergs $\mathrm{cm} \mathrm{s}^{-1}$. Figure 5 illustrates this spectral fit.

Having satisfactorily modeled the soft excess we finally reran the whole broadband model, with the hard X-ray fit parameters (absorption and reflection) now free to vary. We also allowed the abundances of $\mathrm{O}$ and $\mathrm{Ne}$ (responsible for the main peaks at $\sim 0.5$ and $\sim 0.8 \mathrm{keV}$ ) to vary from solar. An important change in this revised fit was a reduction in the cold reflection factor, falling to $R=2 \pm 1$, a consequence of the soft X-ray components also contributing to the observed spectral curvature below $\sim 4 \mathrm{keV}$. The unabsorbed power-law component for this fit is $\sim 1.7 \%$ of the flux of the intrinsic power-law continuum. The resulting final model parameters, with errors, are summarized in Table 2. Figure 6 reproduces the overall input spectrum in this full broadband spectral fit.

Based on the full spectral fit we derive an observed $2-10 \mathrm{keV}$ flux for $2 \mathrm{M} 1049+5837$ of $4.4 \times 10^{-13} \mathrm{ergs} \mathrm{cm}^{-2} \mathrm{~s}^{-1}$. Correcting

\footnotetext{
5 In contrast to Mrk 3, a thermal model cannot be ruled out for 2M1049+5837 given the lower signal-to-noise ratio of the data.
} 


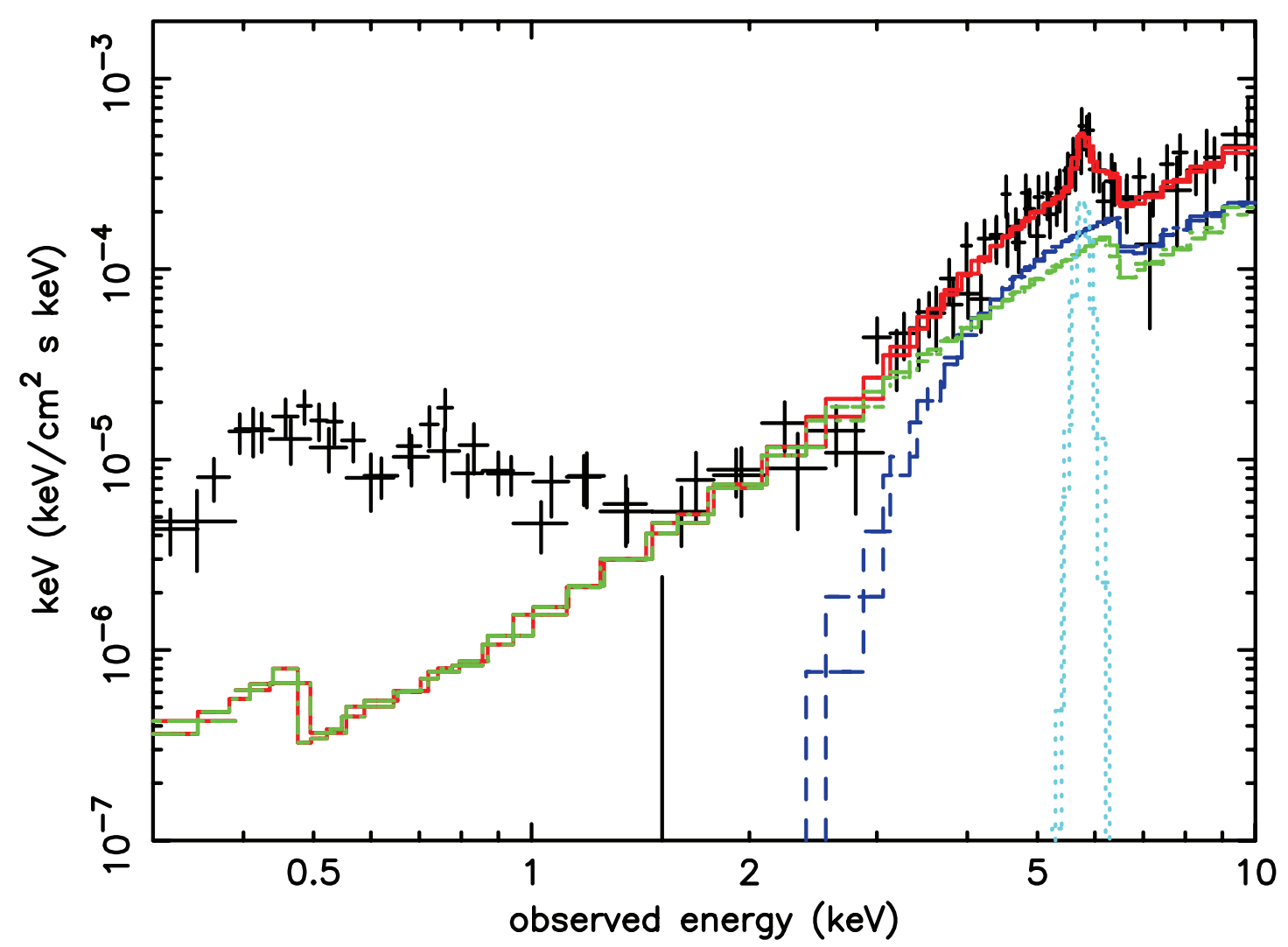

FIG. 3.-Extension of the model fit shown in Fig. 2 to $0.3 \mathrm{keV}$, showing a strong and structured soft excess.

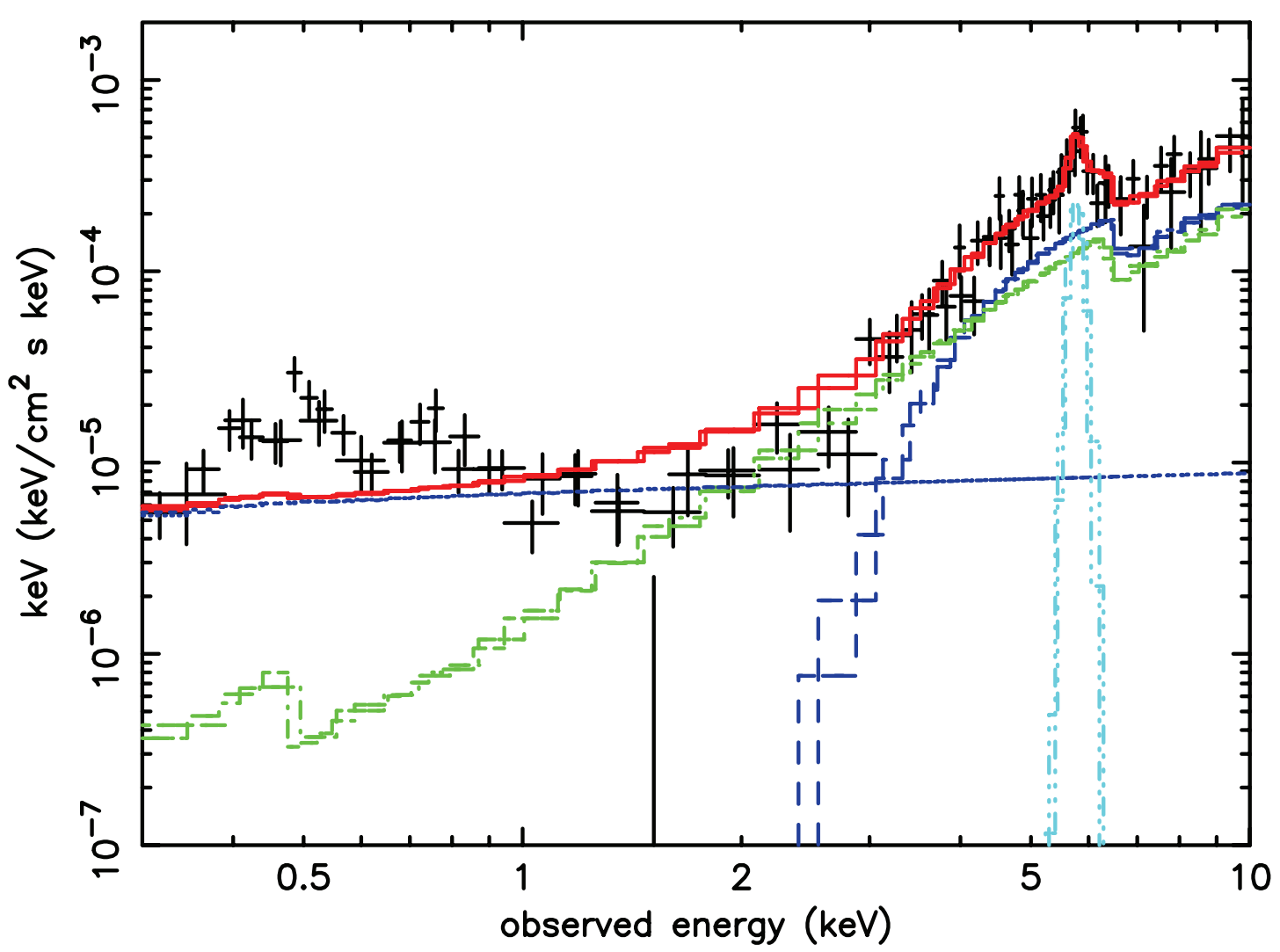

FIG. 4.- Same as Fig. 3, but with the addition of an unabsorbed power-law component (dark blue) to model a fraction of the intrinsic continuum scattered into the line of sight by an extended region of ionized gas. 


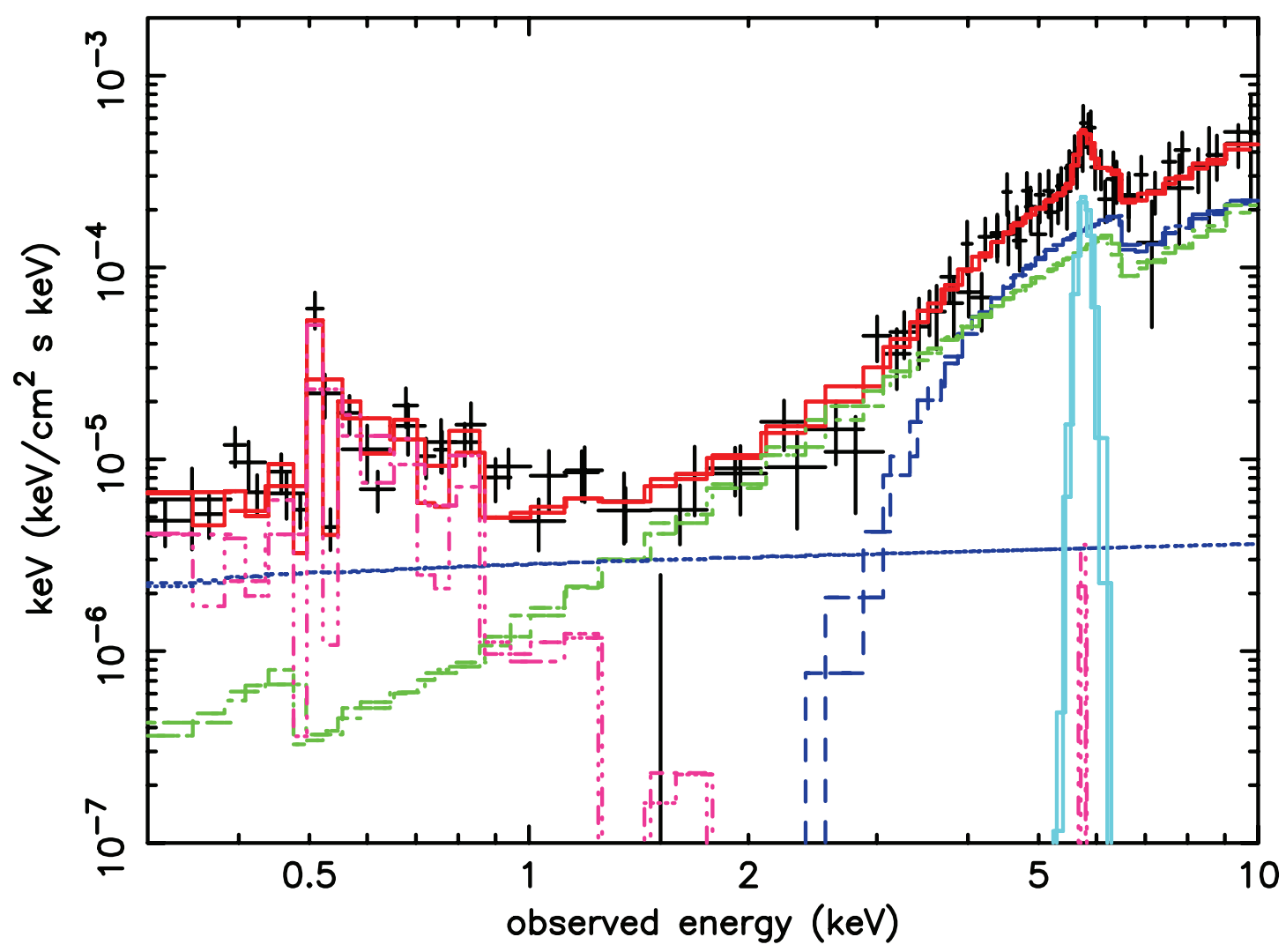

FIG. 5. - Same as Fig. 4, but with the further addition of the emission spectrum of photoionized gas (magenta) to better model the observed structure in the soft X-ray spectrum of $2 \mathrm{M} 1049+5837$.

for the measured absorption the $2-10 \mathrm{keV}$ flux increases to $9.5 \times$ $10^{-13} \mathrm{ergs} \mathrm{cm}^{-2} \mathrm{~s}^{-1}$, corresponding to an unabsorbed luminosity of $3 \times 10^{43} \mathrm{ergs} \mathrm{s}^{-1}$, a factor of $\sim 2$ greater than Mrk 3 (Pounds $\&$ Page 2005). The soft excess contains an observed flux (0.3$2 \mathrm{keV}$ ) of $3.7 \times 10^{-14} \mathrm{ergs} \mathrm{cm}^{-2} \mathrm{~s}^{-1}$, with a soft X-ray luminosity in the emission-line spectrum of $\sim 6 \times 10^{41} \mathrm{ergs} \mathrm{s}^{-1}$. From the absorption-corrected 2-10 keV luminosity of $3 \times 10^{43} \mathrm{ergs} \mathrm{s}^{-1}$ we estimate a bolometric luminosity for $2 \mathrm{M} 1049+5837$ of $6 \times$ $10^{44}$ ergs s $^{-1}$ (Marconi et al. 2004).

\section{DISCUSSION}

\subsection{The Extremely Hard 2-10 keV Spectrum}

Our analysis of the EPIC data of 2M1049+5837 finds the extremely hard 2-10 keV spectrum to be well modeled by an ab- sorbed power law, together with strong reflection from cold matter. This description is similar to that found for the Seyfert 2 galaxy Mrk 3, one of the brightest AGNs in the hard X-ray sky (Pounds \& Page 2005). Although not so well constrained in the case of $2 \mathrm{M} 1049+5837$, the intrinsic power-law continuum is again probably typical of a Seyfert 1 nucleus. With regard to the distribution of the cold matter, it is significant that a good spectral fit at $2-10 \mathrm{keV}$ could not be obtained with the same absorber applied to both the intrinsic power law and the reflected component, implying the reflecting material lies mainly outside the line of sight to the absorbed nucleus.

Identification of the strong emission line at $\sim 6.4 \mathrm{keV}$ (in the AGN rest frame) with fluorescent $\mathrm{Fe} \mathrm{K} \alpha$ emission from cold matter is fully consistent with the lower continuum reflection $(R \sim 2)$ in the broadband spectral fit, with the increased EW (line

TABLE 2

2MASS J1049+5837: XMM-Newton X-Ray Spectral Parameters ( pn CAmera)

\begin{tabular}{|c|c|c|c|c|c|c|c|}
\hline Component & $\begin{array}{c}N_{\mathrm{H}} \\
\left(10^{21} \mathrm{~cm}^{-2}\right)\end{array}$ & Parameter & $\mathrm{EW}(\mathrm{Fe} \mathrm{K} \alpha) / \mathrm{eV}$ & $\chi^{2} / \mathrm{dof}$ & Norm. $^{\text {a }}$ & Flux $^{b}$ & Luminosity $^{\mathrm{c}}$ \\
\hline Power law ........................ & 340 & $\Gamma: 1.8$ & $\cdots$ & $\ldots$ & $25 \pm 5$ & $\ldots$ & $\ldots$ \\
\hline Scattered $\mathrm{PL}^{\mathrm{d}} \ldots \ldots \ldots \ldots$ & 0 & $\Gamma: 1.8$ & $\ldots$ & $\ldots$ & $0.43 \pm 0.25$ & $\ldots$ & 0.05 \\
\hline Reflection ........................ & $\sim 10$ & $R: 2 \pm 1$ & $920 \pm 300^{\mathrm{e}}$ & $\ldots$ & $\ldots$ & & \\
\hline Soft Excess.................... & $\ldots$ & $\xi: 25 \pm 15^{\mathrm{f}}$ & $\ldots$ & $\ldots$ & $\ldots$ & 0.37 & 0.03 \\
\hline Total .................................. & $\ldots$ & $\ldots$ & $\ldots$ & $76 / 75$ & $\ldots$ & 9.5 & 3 \\
\hline
\end{tabular}

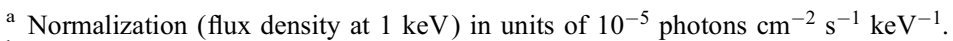

b Absorption-corrected, broadband $\left(2-10 \mathrm{keV}\right.$ ) flux in units of $10^{-13} \mathrm{ergs} \mathrm{cm}^{-2} \mathrm{~s}^{-1}$ (energy band for soft excess is $0.3-2 \mathrm{keV}$ ).

c Absorption-corrected, broadband (2-10 keV) luminosity in units of $10^{43} \mathrm{ergs} \mathrm{s}^{-1}$ (energy band for soft excess is $0.3-2 \mathrm{keV}$ ).

d The best fit also includes a second, unabsorbed power law with the same power-law index at $1.7 \%$ of the intrinsic continuum representing nuclear light scattered off material surrounding the nucleus.

e $\mathrm{Fe} \alpha$ line energy $=6.44 \pm 0.05 \mathrm{keV}$.

${ }_{\mathrm{f}}^{\mathrm{f}}$ The soft excess is modeled by line emission from photoionized gas using an XSTAR grid with best-fit parameter $\xi\left(=L / n r^{2}\right) \mathrm{ergs} \mathrm{cm} \mathrm{s}^{-1}$ and emission measure $\sim 3 \times 10^{64} \mathrm{~cm}^{-3}$. 


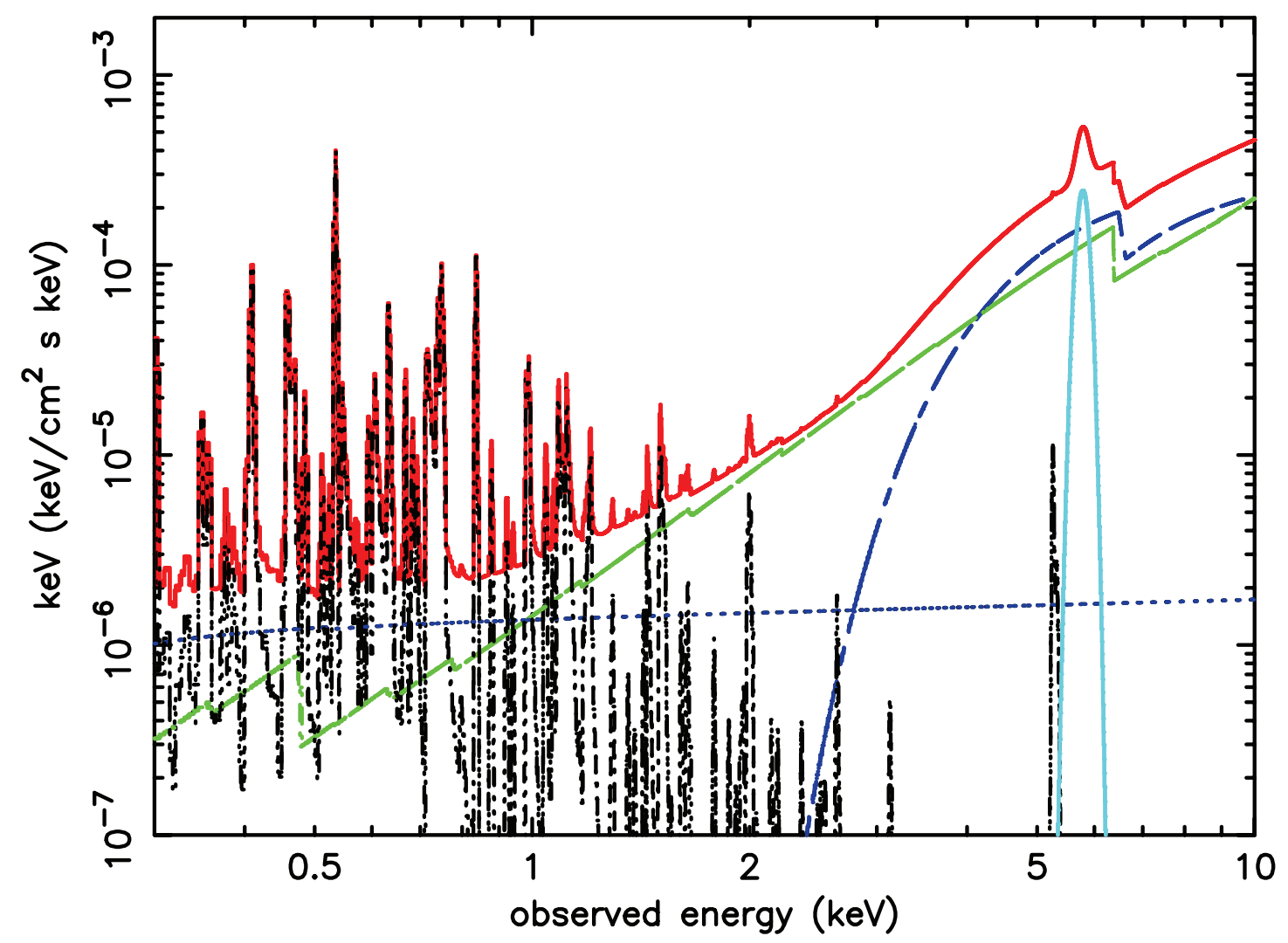

FIG. 6.- Illustration of the final model fit to the $0.3-10 \mathrm{keV}$ EPIC data for $2 \mathrm{M} 1049+5837$. The small redshift of the photoionized emission spectrum relative to the $2 \mathrm{M} 1049+5837$ rest frame is not considered to be real.

to reflected continuum) of $920 \pm 300 \mathrm{eV}$ now in good agreement with the theoretical value (Matt et al. 1997).

As in Mrk 3 the strong reflection continuum and $\mathrm{Fe} \mathrm{K} \alpha$ line are consistent with an origin in the exposed far inner wall of a torus, the near side of which could then form the absorbing barrier to the primary power-law source.

\subsection{Evidence for an Extended Region of Photoionized Gas}

Although the EPIC spectrum of $2 \mathrm{M} 1049+5837$ is less well constrained than that of Mrk 3 (the latter source being more than an order of magnitude brighter and the $X M M-N e w t o n$ observation 3 times longer), we have seen that the similar form of the fluxed spectra gave a useful lead to modeling the hard X-ray spectrum of $2 \mathrm{M} 1049+5837$. In modeling the soft excess in 2M1049+5837 we have also followed the Mrk 3 analysis, obtaining a good statistical fit to the EPIC data with a combination of a weak, unabsorbed power-law continuum and an emission-line spectrum modeled by a photoionized gas in XSTAR. Although the EPIC soft X-ray data are less well determined in $2 \mathrm{M} 1049+5837$, and we have no useful higher resolution spectra from the Reflection Grating Spectrometer (RGS), it seems reasonable, given the similar broad spectral features and relative strength of the soft excess in the two cases, to consider the soft X-ray components of $2 \mathrm{M} 1049+5837$ in the same way as was established for Mrk 3.

In the case of Mrk 3 the RGS spectrum was found (Pounds \& Page 2005) to be dominated by narrow emission lines and radiative recombination continua (RRC) of highly ionized gas. The agreement with the Chandra observation reported by Sako et al. (2000) was good, consistent with the conclusion of the latter authors that both photoionization/recombination and photoexcitation/ radiative emission are important in the soft X-ray spectrum of Mrk 3. The Mrk 3 EPIC fit included a power-law component of $\sim 1 \%$ of the flux in the intrinsic power law, similar to the $\sim 1.7 \%$ in the present modeling of $2 \mathrm{M} 1049+5837$. Assuming electron scattering of the primary continuum in an ionized outflow is responsible for the unabsorbed power-law component, we then have the product $C \tau \sim 0.017$, where $C$ is the covering factor of the ionized outflow and $\tau$ is its optical depth in the line of sight.

Further information on the ionized gas can be obtained from the XSTAR modeling of the emission-line spectrum, which yielded an emission measure of order $3 \times 10^{64} \mathrm{~cm}^{-3}$ (Table 2). The extent of the emission region is not directly constrained by the line intensities or ionization parameter, but given the type 1.8 nature of $2 \mathrm{M} 1049+5837$ we assume a minimum observable radius $r \sim 1 \mathrm{pc}\left(3 \times 10^{18} \mathrm{~cm}\right)$. Although the optically extended structures in $2 \mathrm{M} 1049+5837$ extend beyond $1 \mathrm{kpc}(\S 3.3)$, the $n_{e}^{2}$ radial dependence of any soft X-ray emission would make an expanding ionized outflow "core-bright" so that the majority of the X-ray flux would likely originate close to the nucleus. Assuming the emitting volume to be approximated by a cylinder of radius $r$ and length $30 r$, a uniform density gas with the observed emission measure would then have $n_{e} \sim 3 \times 10^{3} \mathrm{~cm}^{-3}$. The ionization parameter $\xi\left(=L / n r^{2}\right)$ allows a consistency check on that very simple estimate, where $L$ is the (unabsorbed) X-ray continuum flux irradiating the outflow. The EPIC spectral fit found $L(\gtrsim 0.3 \mathrm{keV}) \sim$ $5 \times 10^{43} \mathrm{ergs} \mathrm{s}^{-1}$, yielding the XSTAR model ionization parameter, $\xi \sim 25$ ergs $\mathrm{cm} \mathrm{s}^{-1}$, at a radius of $r \sim 10 \mathrm{pc}$. In this case $\mathrm{O}$ VII and $\mathrm{Ne}$ IX would both be prominent (as indicated by the observed features at $\sim 0.5$ and $\sim 0.8 \mathrm{keV}$ ). To be consistent with the optical data, this region must not overproduce the narrow, optical $\mathrm{H} \alpha$ emission line, which thus provides a check on the electron density in this material. The observed flux of the $\mathrm{H} \alpha$ line is $4.6 \times 10^{-14}$ ergs $\mathrm{cm}^{-2} \mathrm{~s}^{-1}$, with $15 \%-25 \%$ estimated to be narrow. This leads to an estimated luminosity in the narrow $\mathrm{H} \alpha \sim 3 \times 10^{41} \mathrm{ergs} \mathrm{s}^{-1}$ and a resulting emission measure of $2 \times 10^{65} \mathrm{~cm}^{-3}$. This is an order of magnitude greater than the 
estimate for the X-ray-emitting region given above, implying that the optical line originates in a larger region, as expected from the optical data.

The [O III] narrow emission line generally scales well with the X-ray luminosity of an AGN when both are substantially unobscured. The [O III] luminosity for $2 \mathrm{M} 1049+5837$ is $4.5 \times$ $10^{41} \mathrm{ergs} \mathrm{s}^{-1}$ and "not particularly weak" among 2MASS QSOs (Schmidt et al. 2007). Using the ratio deduced by Mulchaey et al. (1994), $\log \left(F_{[\mathrm{O} \text { III }} / F_{\mathrm{X}}\right) \sim-1.9$, yields a $(2-10 \mathrm{keV})$ X-ray luminosity of $3.5 \times 10^{43} \mathrm{ergs} \mathrm{s}^{-1}$. The close agreement with the absorption-corrected value in the $X M M-N e w t o n$ data suggests that the view of the narrow emission line region (NELR), both toward the AGN nucleus and us, is largely unobscured in $2 \mathrm{M} 1049+5837$.

\subsection{The Complex Optical Properties of $2 M 1049+5837$}

As part of their program to observe red, 2MASS quasars, Schmidt et al. (2007) obtained optical spectropolarimetry of $2 \mathrm{M} 1049+5837$ which showed very high polarization $(8 \%-30 \%)$ in two distinct components, one red and one blue, most likely originating in different physical regions of the source. Further investigation via deep HST imaging suggests that the red component originates in scattering of the nuclear emission off material in a bipolar cone, as typically observed in edge-on quasars/AGNs, and probably related to the NELR in this quasar. The red color of both the "fringe" and the polarized light suggests some obscuration of the nuclear light en route to or within the scattering region and/or in between the scattering region and us. The second, weaker component is very blue, dominating the blue end of the optical spectrum, and seems likely to originate in a "fan" of blue emitting material visible in the $H S T$ data and extending at least $\sim 1.6-2.8 \mathrm{kpc}$ from the nucleus. A third scattered light component that appears in the [O III] emission lines, with a position angle slightly different from the red component and a lower polarization level, may originate in dust which is partially segregated from the ionized gas in the red fringe (Schmidt et al. 2007).

Those authors suggest that $2 \mathrm{M} 1049+5837$, along with other highly polarized type 1 quasars/AGNs, has more dust than a typical AGN/quasar and that this dust provides obscuration and scattering of the nuclear light which, when combined, allow the detection of weaker nuclear emission features than would normally be visible. The scattering of significant broad-line emission would then change the optical type such that a source could appear as of intermediate type, in this case type 1.8 , even when viewed near edge-on.

\subsection{An X-Ray Comparison with Mrk 3}

We have found $2 \mathrm{M} 1049+5837$ to be a factor of $\sim 2$ more luminous than the bright, nearby Seyfert 2 galaxy Mrk 3, and to have a similarly unusual X-ray spectrum. In both cases, spectral analysis finds the extremely hard $2-10 \mathrm{keV}$ spectrum to be the sum of an absorbed nuclear continuum plus a strong reflection component and $\mathrm{Fe} \mathrm{K}$ emission line from cold, dense matter lying outside the main line-of-sight absorption. Detection of the direct nuclear X-ray continuum shows the intervening absorber to be sub-Compton-thick in 2M1049+5837 and Mrk 3, suggesting a lower inclination than for classical type 2 AGNs such as NGC 1068. Depression of the intrinsic X-ray continuum in 2M1049+5837, as in Mrk 3, allows scattering and emission from an external region of ionized gas to be readily detected, as is often the case in type 2 AGNs (Guainazzi et al. 2005). The relative strength of both soft X-ray components are again very similar in 2M1049+5837 and Mrk 3, supporting the case for similar geom- etries. The scattered continuum fluxes indicate an effective covering factor $C \tau \sim 0.01-0.02$, while the soft X-ray luminosity of the ionized gas is $\sim 1 \%-2 \%$ of the absorption-corrected $0.3-$ $10 \mathrm{keV}$ luminosity in both 2M1049+5837 and Mrk 3 (Pounds \& Page 2005). As noted in Pounds \& Wilkes (2007), the comparable soft X-ray emission luminosities in several type 1 AGNs are an order of magnitude higher, suggesting that the innermost region of a core-bright ionized outflow is obscured in Seyfert 2 galaxies, and in 2M1049+5837. The lack of similar obscuration for [O III] ( $\S 3.2)$ implies that the NELR and X-ray-emitting regions are largely not cospatial in $2 \mathrm{M} 1049+5837$.

The full extent of the unobscured soft X-ray emission region in $2 \mathrm{M} 1049+5837$ is unknown, but again some insight might be obtained from Mrk 3, where Chandra grating spectroscopy (Sako et al. 2000) showed X-ray emission extending over $\sim 600 \mathrm{pc}$, coincident with the [O III]-emitting region and with flux consistent with the soft excess flux estimated by Pounds \& Page (2005). This spatial scale is consistent with the inner regions of the red fringe. Whether the blue fan contributes significantly to the soft X-ray emission from 2M1049+5837 would depend on the outlying gas retaining a sufficiently high ionization state, in turn depending on the density. We look forward to more sensitive X-ray imaging, which will allow such ionized matter to be mapped.

\subsection{The Relation between Optical and X-Ray Emission Components}

Both X-ray and optical observations of 2M1049+5837 are complex and apparently dominated by scattered light from the central continuum source. Strong obscuration of the nucleus allows weaker components to be visible in both wave bands. Unfortunately, no spatial information is available for the X-ray components of $2 \mathrm{M} 1049+5837$, although it seems likely that the cold reflection components (continuum and $\mathrm{Fe} \mathrm{K}$ fluorescent line) arise from cold, dense matter associated with the nuclear obscuration, perhaps the putative torus or dense inner regions of a disk + wind.

The optical data indicate strong obscuration of the direct nuclear light with reddening of $A_{V} \gtrsim 5$ (Schmidt et al. 2007). The gaseous column density to the nucleus indicated by the X-ray obscuration is $N_{\mathrm{H}} \sim 4 \times 10^{23}$ atoms $\mathrm{cm}^{-2}$, a factor of $\sim 10$ higher than the lower limit dust column for a Galactic gas-to-dust ratio. This level of discrepancy between the optical and X-ray column densities is not unusual observationally (Maiolino et al. 2001) or necessarily unexpected near to a quasar nucleus (e.g., in a wind above a disk; Königl \& Kartje 1994) where dust is unlikely to survive. The obscuration, while high, remains Compton thin, intermediate between classical type 2 AGNs (generally Compton thick) and the low obscuration generally seen in type 1 sources.

\subsubsection{The Reflected X-Ray Component}

The X-ray spectral fitting requires the reflected continuum component to be significantly less obscured than the central source. This suggests a specific geometry in the standard AGN unification model, in which our line of sight passes through the edge of the torus or the inner wind above an accretion disk, attenuating our view of the active nucleus, while the nuclear light reflected from the cold $\left(<10^{4} \mathrm{~K}\right)$, obscuring material on the far side of the nucleus is clearly visible (illustrated schematically in Fig. 7). The geometry thus seems to be very similar to that of Mrk 3 (Pounds $\&$ Page 2005), in which our view is deduced to be "over-theedge" of the obscuring material, as opposed to NGC 1068, in which the reflection component is significantly weaker $(R \sim 0.11$; Pounds \& Vaughan 2006), the obscuration Compton thick, and the viewing angle probably closer to edge-on. 


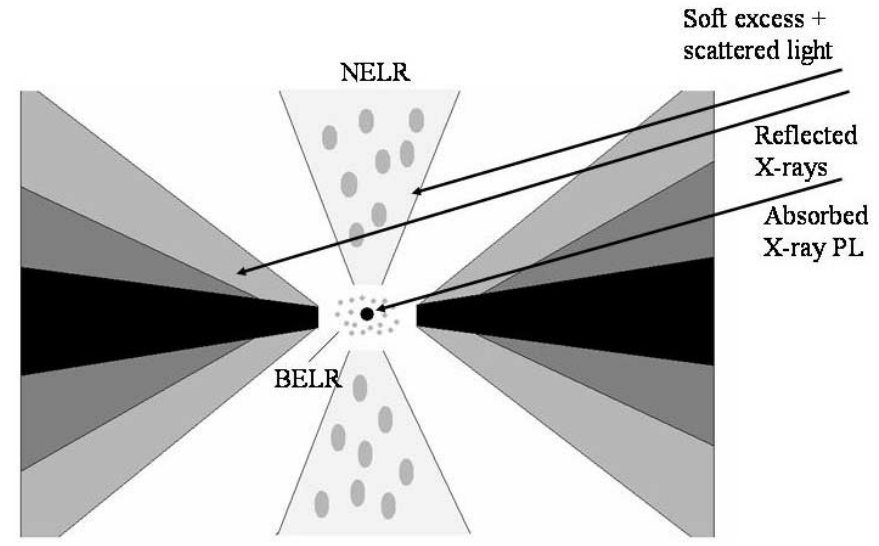

FIG. 7.-Schematic illustration of the geometry for the inner regions of 2M1049+ 5837, showing an accretion disk (black) with a wind/atmosphere (shaded) in which the density decreases away from the disk. The arrows indicate our likely lines of sight to the various emitting regions based on the X-ray data analysis. These provide strong obscuration to the AGN nuclear power law and weaker obscuration of the reflected component. The soft excess is partially covered due to the fairly high inclination of the system, while the NELR is largely unobscured.

\subsubsection{The Soft X-Ray Excess}

The soft X-ray excess shows spectral structure indicative of emission from photoionized material, as is typically seen in these sources (Pounds \& Page 2005; Sako et al. 2000; Levenson et al. 2006; Pounds \& Vaughan 2006). If the soft X-ray emission arises in an ionized outflow driven off the accretion disk or torus wall, then the radial emissivity will depend strongly on both density and ionization gradients. Expansion with a constant velocity would give a core-bright emission (as indicated perhaps by the relative soft X-ray luminosities in type 2 and type 1 AGNs noted earlier; $\S 3.4)$. Extended soft X-ray emission ( $\sim 500 \mathrm{pc})$, which is spatially coincident with optical [O III] emission, has been reported in several bright Seyfert 2 galaxies (e.g., NGC 1068, Mrk 3; Sako et al. 2000; Bianchi et al. 2006), implying an constant ionization parameter with radius. The optically extended regions in the HST imaging for $2 \mathrm{M} 1049+5837$ are larger, $\sim 1-3 \mathrm{kpc}$, although there is no information on the extent of the [O III] emission. 2M1049+ 5837 is at higher redshift and luminosity, which could perhaps result in more extended emitting regions. Below we consider the possibility of overlap between the extended optical and the X-ray scattering/emitting regions.

The blue fan appears to have a clear view of the nucleus based on its color. If we approximate this region as a cylinder $1 \mathrm{kpc}$ from the nucleus with inner radius $0.5 \mathrm{kpc}$ and length $\sim 3 \mathrm{kpc}$ (Schmidt et al. 2007), and use the emission measure from the $X M M-N e w t o n$ spectral fit $\left(\mathrm{EM} \sim 3 \times 10^{64} \mathrm{~cm}^{-3}\right)$, we deduce a mean electron density, $n_{e} \sim 0.7 \mathrm{~cm}^{-3}$ for a uniform medium, in order to produce the observed soft X-ray flux. Again using the ionization parameter as a check, we find $\xi \sim 25 \mathrm{ergs} \mathrm{cm} \mathrm{s}^{-1}$ at a radius of $500 \mathrm{pc}$, roughly consistent with the assumed inner extent of the blue fan. It is also consistent with the inner regions of the red fringe, which is $\lesssim 2 \mathrm{kpc}$ in extent but covers a larger, bipolar region. A similar calculation applied to the red fringe suggests $n_{e} \sim 0.4 \mathrm{~cm}^{-3}$, comparable because the volume of the bipolar region is similar (assuming a cone of emission extending from 1 to $2 \mathrm{kpc}$ in opposing directions with opening angle $\sim 60^{\circ}$ ).

We have no direct constraints on the size of the X-ray-emitting region. For a uniform expanding outflow, where $n_{e} \propto r^{-2}$, the same ionization parameter can be obtained over a wide range of radii. Thus, we can obtain the same observed X-ray emission from a region of size $\sim 1 \mathrm{kpc}$ with $n_{e} \sim 0.7 \mathrm{~cm}^{-3}$, with size $\sim 100 \mathrm{pc}$ with $n_{e} \sim 20 \mathrm{~cm}^{-3}$, or 10 pc with $n_{e} \sim 3 \times 10^{3} \mathrm{~cm}^{-3}(\S 3.3)$. The extended X-ray and [O III]-emitting regions seen in lower luminosity Seyfert galaxies (Bianchi et al. 2006) are $\sim 100-500 \mathrm{pc}$, consistent with the inner regions of the red fringe or the blue fan. However, our conclusion earlier that the soft excess X-ray emission is partially obscured, based on its low flux relative to the harder power law, favors a core-bright emission distribution in which much of the soft excess is obscured while the outer parts, perhaps extending outward as far as the inner regions of the red fringe and/or blue fan, are the only areas visible to us in this "over-the-edge" AGN.

A search for extended X-ray emission with Chandra would help to constrain the location of the soft excess X-ray emission and thus the physical conditions in the region. Meanwhile, we do not detect any variability in the soft X-ray excess over the 2.5 years between the Chandra (2003 March 16) and XMM-Newton (2005 October 10) observations, which provides weak (given the errors) support for emission from an extended region. No information on the extent of the [O III]-emitting region is available, but the "normal" [O III] and $\mathrm{H} \alpha$ line strengths ( $§ 3.2$ ) imply that the NELR is larger and less obscured.

\subsubsection{The X-Ray Scattered Light Component}

The XMM-Newton spectral fit also includes an unobscured, scattered X-ray power-law component at a flux level of $\sim 1.7 \%$ of the intrinsic nuclear power-law component and contributing $\sim 50 \%$ of the soft X-ray flux of $2 \mathrm{M} 1049+5837$. A scattered continuum component often accompanies the RRC spectrum. A weak component is seen in Circinus $(\$ 30 \%$ of the soft X-ray flux; Sambruna et al. 2001$)$, Mrk $3(\sim 0.5 \%$ of the total X-ray luminosity; Sako et al. 2000), and ESO 428-G14 ( $<30 \%$ of the soft flux; Levenson et al. 2006), whereas none is detected in NGC 1068 (Kinkhabwala et al. 2002).

Assuming electron scattering and adopting a covering factor, $C \sim 0.16$ based on the geometry of the blue fan adopted earlier, leads to an estimate of the electron column density, $N_{e} \sim 1.5 \times$ $10^{23} \mathrm{~cm}^{-2}$ and a corresponding average electron density, $n_{e} \sim$ $17 \mathrm{~cm}^{-3}$. This is comparable with that deduced for the soft excess emitting region if its physical size is $\sim 100 \mathrm{pc}$.

From the optical polarization data and an assumed geometry, it is possible to obtain a rough estimate of the scattering column density for the blue fan. The polarized flux at $4000 \AA$ is $\sim 0.5 \times 10^{-17} \mathrm{ergs} \mathrm{cm}^{-2} \mathrm{~s}^{-1} \AA^{-1}$, and the unobscured flux of the AGN is estimated at $\sim 2 \times 10^{-15} \mathrm{ergs} \mathrm{cm}^{-2} \mathrm{~s}^{-1} \AA^{-1}$ using the identical redshift, type 1 QSO PG $1552+085$ as a guide (Schmidt et al. 2007). The blue fan geometry assumed above implies that the scattering material subtends a $60^{\circ}$ solid angle on the nucleus, which leads to an estimate of the scattering optical depth $\tau \sim 0.015$ (with large uncertainties). In this case scattering by dust particles rather than electrons is inferred. Adopting a mean dust scattering cross section, $\sigma_{d} \sim 1 \times 10^{-12} \mathrm{~cm}^{2}$ (White 1979), leads to an estimate of the dust column density, $N_{d} \sim 2 \times$ $10^{10} \mathrm{~cm}^{-2}$. Assuming a region size $3 \mathrm{kpc}$ (blue fan, as earlier) and $\rho_{d} / \rho_{e} \sim 0.01$ implies a mean electron density, $n_{e} \sim 0.1 \mathrm{~cm}^{-3}$ (adopting a dust grain size $0.1 \mu \mathrm{m}$ and density $1 \mathrm{~g} \mathrm{~cm}^{-3}$ ). Although highly uncertain, this is 100 times lower than that deduced for the X-ray scattered continuum using the same region geometry.

Thus, for an origin in the larger blue fan region, much of the X-ray scattering must occur in denser material, possibly in clumps within the blue fan similar to those seen in NGC 1068 
(Kinkhabwala et al. 2002). Alternatively the X-ray scattering may occur closer to the AGNs where the electron density is likely to be higher.

\subsubsection{Structure of the Inner Regions}

While a firm conclusion cannot be reached, it seems most likely that the observed soft X-ray excess and scattered X-ray continuum originate closer to the AGNs or from the inner part $\left(n_{e} \sim\right.$ $20 \mathrm{~cm}^{-3}, \sim 100-500 \mathrm{pc}$ ) of the extended optical regions which are responsible for the blue and red scattered optical light. This implies a spatial scale similar to observations in lower luminosity, nearby Seyfert galaxies. Deep X-ray imaging of objects such as $2 \mathrm{M} 1049+5837$ will in future provide a vital complement to optical/IR images and spectra in revealing the structure and dynamics of the complex circumnuclear matter.

The most likely geometry of $2 \mathrm{M} 1049+5837$ is summarized in Figure 7, showing an intermediate line of sight in which the nuclear light is viewed through a fairly dense region close to the surface of the accretion disk/torus and so is heavily obscured. The reflected component is more clearly visible as our line of sight passes farther above the disk/torus where the wind/atmosphere is less dense. The soft excess originates in material above and below the disk (perhaps the inner parts of the NELR/red fringe). This region is largely obscured from view by the outer parts of the wind (being more affected than the hard, reflected X-ray light). The scattered power law may originate in the same/similar material or in denser clumps of material embedded within the lower density NELR. A number of models include lower density obscuring material surrounding an accretion disk, either through a radiatively driven wind (Königl \& Kartje 1994; Elvis 2000) or a radiation pressure support, starburst disk (Thompson et al. 2005), which would allow for such lines of sight.

\section{CONCLUSIONS}

XMM-Newton observations of the red quasar $2 \mathrm{M} 1049+5837$ reveal an unusually hard $(\Gamma \sim-0.6)$ and highly complex X-ray spectrum, reinforcing our earlier conclusion (Wilkes et al. 2005) that low signal-to-noise ratio X-ray spectra can be very misleading. The X-ray spectral fit (Table 2) requires the following components:
1. An absorbed, close to Compton-thick, power law, $N_{\mathrm{H}} \sim$ $3.4 \times 10^{23} \mathrm{~cm}^{-2}$;

2. an unabsorbed, strong reflection component;

3. a strong soft excess, revealed by the strong absorption of the direct AGN light at low energies, but whose low relative strength implies partial covering and an intermediate line of sight; and

4. an unobscured X-ray power law presumed to be scattered into our line of sight by material outside the AGN nucleus.

The combination of reflection and obscured power law required to explain the hard X-ray spectrum implies an unusual balance between the flux from the nucleus and its reflection. This is unlikely to be common in AGNs in general, since the emerging spectrum is significantly harder than that of the CXRB.

Although no firm conclusions can be drawn, the X-ray soft excess and scattered $\mathrm{X}$-ray power-law emission likely originate close to the AGN nucleus in material with $n_{e} \gtrsim 10 \mathrm{~cm}^{-3}$, which may extend to overlap with the inner parts of one/both of the extended optical emitting regions (Schmidt et al. 2007) on a scale of $\sim 100-500 \mathrm{pc}$. The NELR itself, which is probably synonymous with the red fringe, is more extended, and our view is largely unobscured. High-resolution X-ray and [O III] optical imaging would allow us to investigate the location of the X-ray-emitting gas and its relation to the optical NELR.

We conclude that the source is viewed at an intermediate line of sight in which the central regions are viewed through an atmosphere/wind above a disk/torus, allowing different amounts of absorption for the different X-ray components. This scenario is consistent with a number of suggested models that involved an accretion disk with a wind (Königl \& Kartje 1994; Elvis 2000) or an atmosphere (Thompson et al. 2005) which provides the obscuration.

We wish to thank Charlotte Garcia for assistance in generating Figure 7. The results reported here are based on observations obtained with $X M M$-Newton, an ESA science mission with instruments and contributions directly funded by ESA Member States and the USA (NASA). The authors wish to thank the SOC and SSC teams for organizing the XMM-Newton observations and initial data reduction. B. J. W. is grateful for the financial support of XMM-Newton GO grant NNG 04GD27G.

\section{REFERENCES}

Arnaud, K. A. 1996, in ASP Conf. Ser. 101, Astronomical Data Analysis Software and Systems V, ed. G. H. Jacoby \& J. Barnes (San Francisco: ASP), 17 Bianchi, S., Guainazzi, M., \& Chiaberge, M. 2006, A\&A, 448, 499

Cutri, R. M., Nelson, B. O., Francis, P. J., \& Smith, P. S. 2002, in IAU Colloq. 184 AGN Surveys, ed. R. F. Green, E. Y. Khachikian, \& D. B. Sanders (ASP Conf. Ser. 284; San Francisco: ASP), 127

Elvis, M. S. 2000, ApJ, 545, 63

Gilli, R., Comastri, A., \& Hasinger, G. 2007, A\&A, 463, 79

Guainazzi, M., Matt, G., \& Perola, G. C. 2005, A\&A, 444, 119

Kallman, T., Liedahl, D. A., Osterheld, A., Goldstein, W., \& Kahn, S. M. 1996, ApJ, 465, 994

Kinkhabwala, A., et al. 2002, ApJ, 575, 732

Königl, A., \& Kartje, J. F. 1994, ApJ, 434, 446

Kuraszkiewicz, J. K., et al. 2008, ApJ, submitted

Levenson, N. A., Heckman, T. A., Krolik, J. H., Weaver, K. A., \& Zycki, P. T. 2006, ApJ, 648, 111

Magdziarz, P., \& Zdziarski, A. A. 1995, MNRAS, 273, 837

Maiolino, R., et al. 2001, A\&A, 365, 28

Marconi, A., Risaliti, G., Gilli, R., Hunt, L. K., Maiolino, R., \& Salvati, M. 2004, MNRAS, 351, 169

Matt, G., Fabian, A. C., \& Reynolds, C. F. 1997, MNRAS, 289, 175

Mulchaey, J. S., Koratkar, A., Ward, M. J., Wilson, A. S., Whittle, M., Antonucci,

R. J., Kinney, A. L., \& Hurt, T. 1994, ApJ, 436, 586

Polletta, M. M., et al. 2006, ApJ, 642, 673

Pounds, K. A., \& Page, K. L. 2005, MNRAS, 360, 1123

Pounds, K. A., \& Vaughan, S. 2006, MNRAS, 368, 707

Pounds, K. A., \& Wilkes, B. J. 2007, MNRAS, 380, 1341

Pounds, K. A., Wilkes, B. J., \& Page, K. L. 2005, MNRAS, 362, 784

Sako, M., Kahn, S. M., Paerels, F., \& Liedahl, D. A. 2000, ApJ, 543, L115

Sambruna, R. M., Netzer, H., Kaspi, S., Brandt, W. N., Chartas, G., Garmire,

G. N., Nousek, J. A., \& Weaver, K. A. 2001, ApJ, 546, L13

Schmidt, G. D., Smith, P. S., Hines, D. C., Tremonti, C. A., \& Low, F. J. 2007, ApJ, 666, 784

Smith, P. S., Schmidt, G. D., Hines, D. C., Cutri, R. M., \& Nelson, B. O. 2002, ApJ, 569, L23

Smith, P. S., Schmidt, G. D., Hines, D. C., \& Folz, C. B. 2003, ApJ, 593, 676

Stark, A. A., Gammie, C. F., Wilson, R. W., Bally, J., Linke, R., Heiles, C., \& Hurwitz, M. 1992, ApJS, 79, 77

Strüder, L., et al. 2001, A\&A, 365, L18

Thompson, T. A., Quataert, E., \& Murray, N. 2005, ApJ, 630, 167

Turner, M. J. L., et al. 2001, A\&A, 365, L27

Ueda, Y., et al. 2007, ApJ, 664, L79

White, R. L. 1979, ApJ, 229, 954

Wilkes, B. J., Pounds, K. A., Schmidt, G. D., Smith, P. S., Cutri, R. M., Ghosh, H., Nelson, B., \& Hines, D. C. 2005, ApJ, 634, 183

Wilkes, B. J., Schmidt, G. D., Cutri, R. M., Ghosh, H., Hines, D. C., Nelson, B., \& Smith, P. S. 2002, ApJ, 564, L65

Winter, L. M., Mushotzy, R. F., Tueller, J., \& Markwardt, C. 2008, ApJ, 674, 686 